\title{
Characterization of LEAFY in Rubus Coreanus and Its Phylogenetic Application in Rubus Species
}

\author{
Dan JIANG ${ }^{1, \#}$, Hao-ru TANG ${ }^{1, \#}$, Yan WANG ${ }^{2}$ and Qing CHEN ${ }^{1, a, *}$
}

${ }^{1}$ College of Horticulture, Sichuan Agricultural University, Chengdu 611130, Sichuan, China

${ }^{2}$ Institute of Pomology and Olericulture, Sichuan Agricultural University, Chengdu 611130, Sichuan, China

\#These authors contributed equally to this work

asupnovel@gmail.com

${ }^{*}$ Corresponding author

Keywords: Rubus, LEAFY, Clone, Phylogenetic application.

\begin{abstract}
Rubus coreanus is endemically distributed in Southwest China and has great potential for Rubus breeding due to its nutritional values and insects and pests' resistance. $L E A F Y$, as a single copy nuclear gene in diploid plants, plays an important role in the taxonomy and phylogeny, containing three exons and two introns. Here we designed new primers to amplify $L E A F Y$ gene fragment based on gene sequences from apple, strawberry, pear and peach. The new designed primers were demonstrated to be applicable for several other Rubus species in Rubus genus. The full length of $L E A F Y$ fragment in $R$. coreanus was $1127 \mathrm{bp}$, including $319 \mathrm{bp}$ at the $3^{\prime}$ end of the second exon, $729 \mathrm{bp}$ of the second intron, and $39 \mathrm{bp}$ from the $5^{\prime}$ end of the third exon. Its GC content was $30 \%$ while AT content counted for $70 \%$. We further reconstructed the phylogenetic tree using several $L E A F Y$ gene from Rosaceae species. These results could provide effective tool to study the phylogenetic relationships within the Rubus genus.
\end{abstract}

\section{Introduction}

The genus Rubus is one of the largest genera in the Rosaceae family, consisting of 750-1000 species in many parts of the world [1,2]. Those Rubus plants used for horticultural plantations are generally named bramble or edible Rubus. Due to its nutritional values and medical uses, Rubus fruits are named Fruits of Life by Americans brambles were recommeded as the third generation fruits by FAO [3]. Until today, brambles have been widely planted in many parts of the world and have increasingly become the most rapidly developing small fruit tree just next to strawberry, blueberries and currants [4].

Rubus coreanus is endemically distributed in Southwest China and holds great potential for Rubus breeding [5, 6]. There existed divergences between taxonomy and phylogeny of Rubus [7], which affected the utilization of these excellent wild resources in breeding. DNA sequence is an ideal method for phylogeny and classification of various taxa in plants [8]. Compared with other sequences, low-copy nuclear genes (LCNG) can provide more informative sites, especially for lower taxonomic levels [9]. $L E A F Y$ plays an important role in the formation of floral meristem [10,11], containing three exons and two introns. Previous study suggested that the second intron of the $L E A F Y$ gene is useful for phylogenetic reconstructions of closely related species [10, $12]$. 
At present, the research on $L E A F Y$ in Rubus, including the function to regulate flowering and the application in phylogeny, are very limited. Yang et al. [13] used the $L E A F Y$ sequence to study the phylogenetic relationship among Korean Rubus. However, when we further verified the sequence, we found that all the reported 'LEAFY' did not match any sequences by the functional detection of $L E A F Y$ nucleic acid sequence from the GenBank database. Thus, we speculated that they did not obtain Rubus $L E A F Y$ gene. In this study, we designed new primers to amplify the $L E A F Y$ gene, and focused on the second intron to reconstructed phylogenetic tree. This study will provide the effective basis for the taxonomy and phylogeny of $R$. coreanus.

\section{Materials and Methods}

\section{Materials}

The genomic DNA of $R$. coreanus, E. coli JM109 competent cells were stored in laboratory and the cloning vector pMD19-T was purchased from Takara Company.

\section{Methods}

Based on the exon sequences of $L F Y$ second intron in apple, strawberry, peach and plum, primers LFY2F: 5'-GGCTGTCGGAGGAGCCRGTG-3', and LFY2R: 5'-CAATGTCCCARCCTTGGCSTGC-3' were designed for amplification. Polymerase chain reaction (PCR) amplification was performed in a total volume of 20 $\mu \mathrm{L}$, containing $2 \mu \mathrm{L}$ of template DNA (10-50 ng), $10 \mu \mathrm{L}$ of Taq Mix, $1 \mu \mathrm{L}$ of each primer $(5 \mu \mathrm{M})$. Conditions for amplification consisted of an initial denaturation at $94{ }^{\circ} \mathrm{C}$ for $3 \mathrm{~min}$, followed by 35 cycles at $94{ }^{\circ} \mathrm{C}$ for $45 \mathrm{~s}$, then at $66{ }^{\circ} \mathrm{C}$ to $58{ }^{\circ} \mathrm{C}$ with $0.5{ }^{\circ} \mathrm{C}$ decreasing per cycle for $1 \mathrm{~min}$ and $72{ }^{\circ} \mathrm{C}$ for $1 \mathrm{~min}$, with a final extension at $10 \mathrm{~min}$.

PCR products were verified by $1 \%$ agarose gel electrophoresis and photographed by EB staining, and purified by TaKaRa MiniBEST Agarose Gel DNA Extraction Kit. The amplicons were further ligated to pMD19-T vector and transformed into JM109 competent cells. A single clone was picked to expand cultivation, and identified by PCR amplification. The positive clones were sequenced by Sangon Biotech company.

The boundaries between exons and introns were determined by alignment Rubus $L E A F Y$ sequence and preservations of the 'GT' and 'AG' at two ends of introns. Sequences were aligned with Muscle, and manually adjusted in the Molecular Evolutionary Genetics Analysis sofaware (MEGA 7.0) with gaps treated as missing data. Some $L E A F Y$ sequences of other Rosaceae species were obtained from GenBank. The minimum evolution tree was reconstructed by MEGA 7.0.

\section{Results}

\section{PCR Amplification}

The newly designed primer successfully amplified a fragment of about $1200 \mathrm{bp}$ (Fig. 1) without any non-specific products. PCR products were purified, cloned and ligated by vector pMD19-T. Then we obtained the true $L E A F Y$ gene sequences after sequencing. The fragment length of the gene was $1127 \mathrm{bp}$ in length, including a fragment of $319 \mathrm{bp}$ at the $3^{\prime}$ end of the second exon, $729 \mathrm{bp}$ of the second intron and a $39 \mathrm{bp}$ fragment from the $5^{\prime}$ end of the third exon. The sequence was characterized by high AT content (70\%), but has high level of similarity to those of reported $L E A F Y$ sequence in other species (Fig. 2). 


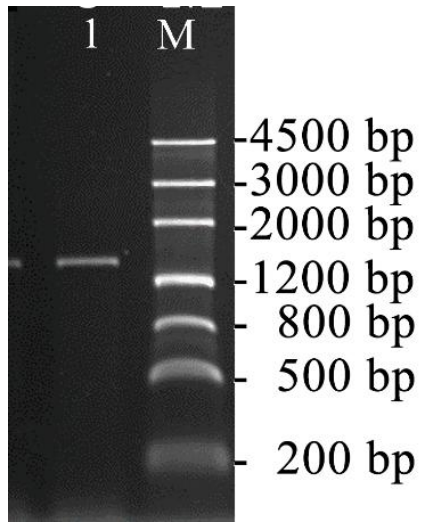

Figure 1. PCR amplification of LEAFY in Rubus coreanus

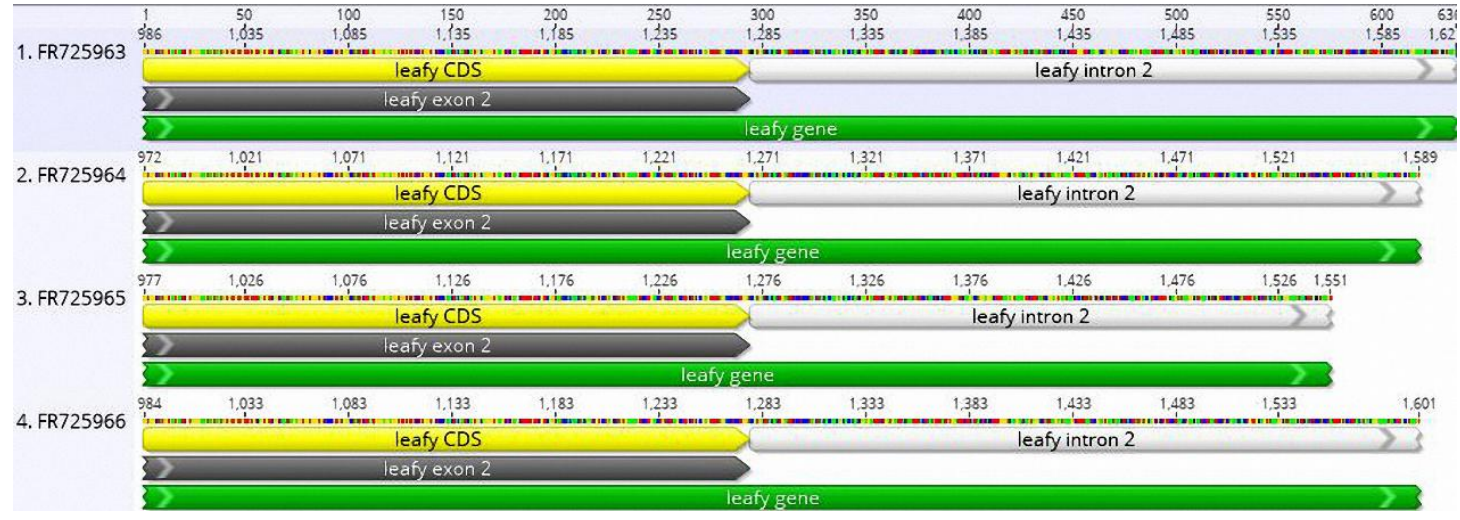

Figure 2. Blast hit results using $L E A F Y$ sequences in the GenBank database

\section{Phylogeny Analysis Using LEAFY}

We downloaded five LEAFY sequences of Malus, Pryus, and Prunus species from NCBI. Addtionally, three deposited Rubus LEAFY genes ( $R$. croceacanthus, $R$. corchorifolius, $R$. parvifolius) from the Korean group were also included. The phylogenetic tree was reconstructed by MEGA 7.0 (Fig. 3). The cloned $L E A F Y$ gene of $R$. coreanus could form a normal evolution tree with other sequence of Rosaceae family. The topology for the other three Rubus species is distantly relate to that of Rubus coreanus, which clearly indicated that our $L E A F Y$ sequence is a completely new sequence.

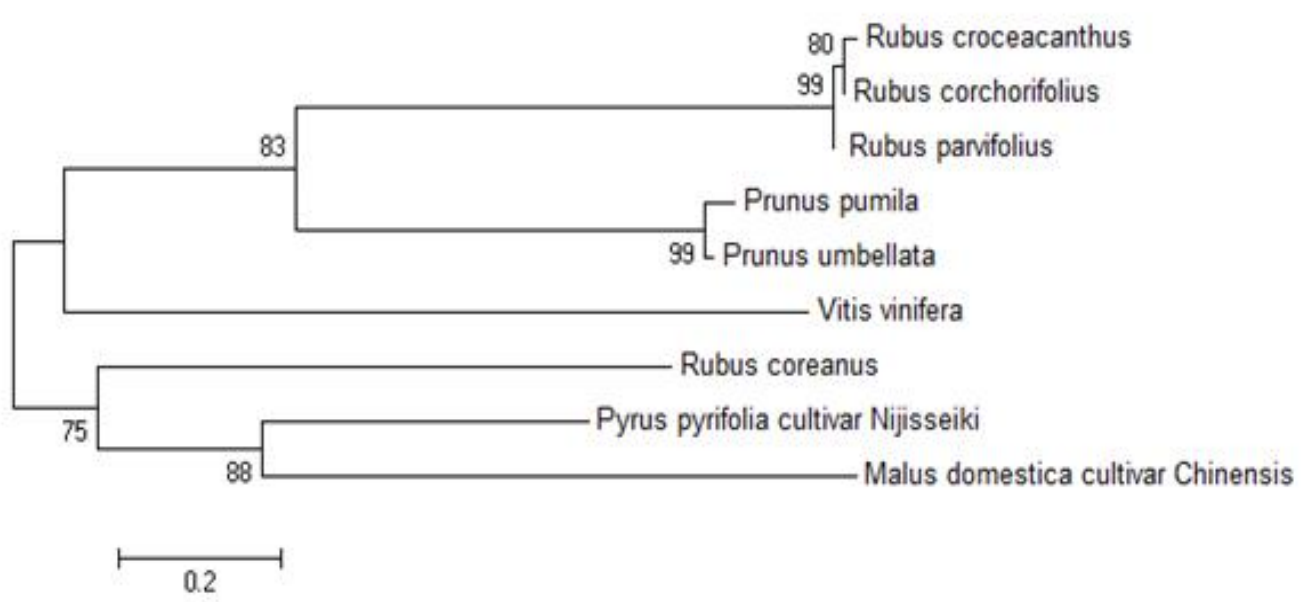

Figure 3. The minimal evolution tree reconstructed by the second intron of $L E A F Y$ from several Rosaceae species 


\section{Application of This LEAFY Primers in other Rubus Species}

To verify the applicability of the designed primers on the Rubus genus, we used seven additional Rubus species for further amplification. As shown in Fig. 4, we obtained the same length $L E A F Y$ gene among different Rubus species. This indicated that the designed primers were feasible in the genus Rubus.

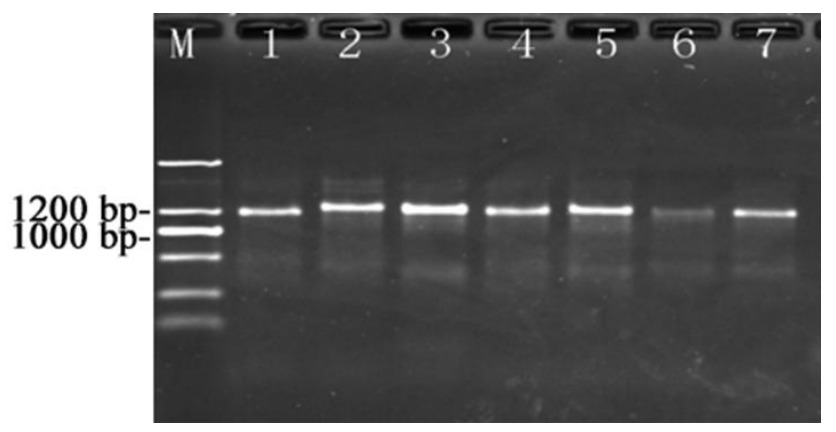

Figure 4. Amplified electrophoresis of $L E A F Y$ among different Rubus species

1: Rubus lambertianus, 2: R. lambertianus var. glandulosus, 3: $R$. stimulans, 4: R. pacificus, 5: $R$. tephrodes, 6: $R$. alceaefolius, 7: $R$. amphidasys.

\section{Discussion}

In this study, we designed new primers for low-copy nuclear $L E A F Y$ gene amplification in $R$. coreanus. We further applied the new designed primers in more Rubus species, indicating that they were feasible within the genus. By cloning and sequence analysis, we obtained the $L E A F Y$ gene fragment with full-length of $1127 \mathrm{bp}$, including $319 \mathrm{bp}$ at the 3 ' end of the second exon, $729 \mathrm{bp}$ of the second intron, and $39 \mathrm{bp}$ from the 5 ' end of the third exon. Its GC content was $30 \%$, while the AT content counted for $70 \%$. Further reconstruction of the phylogenetic tree using several $L E A F Y$ gene from Rosaceae species clearly indicate the fake ' $L E A F Y$ ' genes deposited in NR database. These results could provide effective tool to study the phylogenetic relationships within the Rubus genus.

\section{Acknowledgement}

This research was financially supported by the National Natural Science Foundation of China (Grant Number 31600232).

\section{References}

[1] Z.Z. Qu, Y.W. Sun, Theory of Fruits Species, Beijing: Agricultural Press. 1990, $153-160$.

[2] D.J. Yu, L.D. Lu, C.Z. Gu, Flora of China, Beijing: Science Press. 1980, 37, $10-219$.

[3] G.C. Li, J.M. Su, H.S. Zhang, A brief introduction of raspberry and blackberry varieties, Yantai Fruits 2014, 1, 25-25.

[4] G.J. Bian, Q.Y. Zhou, Principal component analysis of the main economic characters and superior variety selection of raspberry, J. Sichuan Forestry Sci. Technol. 2006, 4(2), 68-71. 
[5] H.M. Jiang, D.H. Shi, X.Q. Wang, X.M. Gou, L.P. Yin, G.Q. Yang, Mechanical shaking extraction and antioxidant activity of anthocyanins from Rubus coreanus miq. fruits, Food Sci. 2014, 35(20), 67-67.

[6] X.R. Wang, H.R. Tang, Q.X. Deng, Advancement in research of genetic diversity of bramble (Rubus L.) and its breeding in China, Acta Hortic. Sinica 2006, 33(1), 190-196.

[7] L. Zhang, X.R. Wang, Y. Wang, Q. Chen, W. He, Research progress of molecular phylogenetic analyses based on DNA sequences data in Rubus L. (Rosaceae), Acta Bot. Boreal. -Occident. Sin. 2014, 34(2), 424-425.

[8] I. Alvarez, F. Wendel, Ribosomal ITS sequence and plants phylogenetic inference, Mole. Phylogenet. Evol. 2003, 29(3), 417-434.

[9] B. Sutherland, Phyligenetics of Rubus ursinus and R. macraei (Rosaceae): evidence of hybrid origin. Bowling Green: Western Kentucky University, 2005.

[10]D.M. Zou, Cloning and expression analysis of floral related genes in Strawberry, Shenyang Agri. Uni. 2012, 9-10.

[11]E.A. Schultz, G.W. Haughn, LEAFY, a homoetic gene that regulate inflorescence development in Arabidopsis, Plant Cell 1992, 3, 771-781.

[12]L. Gong, Characterization and functional analysis of a LEAFY homologous gene in longan (Dimocarpus longan Lour.), Fujian A\&F uni. 2008, 4, 4-4.

[13] J.Y. Yang, H.S. Yoon, J.H. PAK, Phylogeny of korean Rubus based on the second intron of the LEAFY gene. Canadian J. Plant Sci. 2012, 92(3), 461-472. 